\title{
Desenvolvimento e avaliação da eficácia de filmes biodegradáveis de amido de mandioca com nanocelulose como reforço e com extrato de erva-mate como aditivo antioxidante
}

\author{
Development and evaluation of the effectiveness of biodegradable films of cassava starch with \\ nanocelulose as reinforcement and yerba mate extract as an additive antioxidant
}

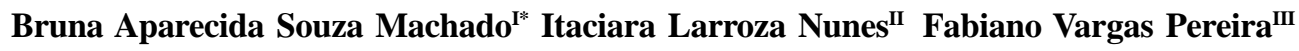 \\ Janice Izabel Druzian ${ }^{\mathrm{I}}$
}

\section{RESUMO}

O objetivo do trabalho foi desenvolver uma embalagem biodegradável utilizando como matriz polimérica o amido de mandioca plastificada com glicerol e reforçada com a incorporação de nanocelulose da fibra de coco, bem como, avaliar o efeito da adição de um aditivo natural (ervamate) nas formulações de nanobiocompósitos com ação antioxidante. Os nanocristais de celulose $(L / D=39)$ foram obtidos por hidrólise ácida com $\mathrm{H}_{2} \mathrm{SO}_{4}$ a $65 \%$. Os filmes foram preparados por casting contendo 4,5 e 6,0\% de amido, 0,5 e $1,5 \%$ de glicerol, $0,3 \%$ de nanocelulose e $20 \%$ de extrato de erva-mate. O armazenamento do azeite de dendê embalado com os filmes contendo o aditivo foi monitorado por 40 dias sob condições de oxidação acelerada $\left(63 \% U R / 30^{\circ} \mathrm{C}\right)$. Constatou-se que, à medida que aumentam as perdas de Polifenóis Totais nos filmes, ocorre um menor aumento do Índice de Peróxidos do produto embalado, demonstrando, assim, que, ao invés do produto, os compostos da embalagem é quem estão sofrendo oxidação. A incorporação de extrato de erva-mate não alterou as propriedades mecânicas e de barreira desses filmes.

Palavras-chave: embalagem ativa, polifenóis, nanobiocompósitos, nanocristais de celulose.

\section{ABSTRACT}

The objective was to develop biodegradable packaging using a polymer matrix as the cassava starch plasticized with glycerol and reinforced with the incorporation of nanocelulose of coconut fiber, as well as to evaluate the effect of the addition of an additive nature (yerba mate) in nanobiocompósitos formulations with antioxidant action. The nanocrystal cellulose $(L / D=39)$ were obtained by acid hydrolysis with $65 \% \mathrm{H}_{2} \mathrm{SO}_{4}$. The films were prepared by casting containing
4.5 and $6.0 \%$ starch, 0.5 and $1.5 \%$ glycerol, $0.3 \%$ nanocelulose and $20 \%$ extract of yerba mate. The palm oil storage packed with films containing the additive was monitored for 40 days under conditions of accelerated oxidation $\left(63 \% U R / 30^{\circ} \mathrm{C}\right)$. It was found that as the losses increase polyphenol films, there is a smaller increase of the peroxide value of the packaged product, thus demonstrating that instead of the product, the compounds of the package's who are suffering oxidation. The incorporation of yerba mate extract did not alter the mechanical and barrier properties of these films.

Key words: active packaging, polyphenols, nanobiocompósitos, cellulose nanocrystals.

\section{INTRODUÇÃO}

Tradicionalmente, os materiais de embalagens têm sido selecionados no sentido de ter a mínima interação com o alimento que acondicionam, constituindo assim barreiras inertes, com função de proteger o produto embalado. Entretanto, na última década, diversos estudos (VEIGA-SANTOS et al., 2005a; GRISI et al., 2008; SILVA, 2009; SOUZA et al., 2011a; MACHADO et al., 2010) têm desenvolvido sistemas de embalagens com o objetivo de interagir com o alimento, utilizando matrizes biodegradáveis e aditivos naturais, planejados para corrigir deficiências das embalagens passivas, e sendo chamadas de embalagens biodegradáveis ativas.

\footnotetext{
'Departamento de Análises Bromatológicas, Faculdade de Farmácia, Universidade Federal da Bahia (UFBA), 40170-115, Salvador, BA, Brasil. E-mail: brunamachado@ufba.br.*Autor para correspondência.

IIDepartamento de Ciência dos Alimentos, Escola de Nutrição, UFBA, Salvador, BA, Brasil.

IIIDepartamento de Química, Universidade Federal de Minas Gerais (UFMG), Belo Horizonte, MG, Brasil.
} 
A biodegradabilidade e o aumento da segurança alimentar são os principais benefícios das embalagens ativamente funcionais, pois contribuem para uma maior preservação do alimento e do meio ambiente. Entretanto, apesar de os filmes de amido possuirem a vantagem de ter caráter biodegradável, apresentam propriedades mecânicas inadequadas, além de uma alta permeabilidade ao vapor de água (VEIGASANTOS et al., 2005a; VEIGA-SANTOS et al., 2005b; MALI et al., 2010), sendo, portanto, impróprios para a comercialização como embalagens. Com o objetivo de melhorar as propriedades mecânicas e de barreiras dos filmes a base de amido e tornar viável a sua comercialização, diversos estudos (LU et al., 2005; CAO et al., 2008; WANG et al., 2010; MACHADO et al., 2010) incorporaram nanopartículas obtidas da celulose de fibras naturais, chamadas de nanocelulose ou nanocristais de celulose.

Os nanocristais de celulose são os domínios cristalinos de fontes celulósicas, obtidos através do processo de hidrólise ácida, possuindo características de elevada rigidez, alta cristalinidade e tamanho nanométrico. Essas nanopartículas, quando isoladas em solução, têm sido incorporadas e avaliadas como material de reforço em matrizes poliméricas, devido ao seu potencial para melhorar as propriedades mecânicas, de barreira, térmicas, ópticas e dielétricas, e, quando incorporadas em matrizes obtidas de fontes renováveis, resultam na formação de nanobiocompósitos totalmente biodegradáveis.

Dentre os diversos tipos de embalagens ativas, as que exercem efeito antioxidante estão entre as mais importantes para a indústria, principalmente para a alimentícia. A oxidação é uma das principais reações de degradação que ocorre nos alimentos, limitando a sua conservação (FUKUMOTO \& MAZZA, 2000; LOULI et al., 2004).

Dentre as diversas fontes naturais de polifenóis, destaca-se a erva-mate (Ilex paraguariensis Saint Hílare), que apresenta em sua composição química compostos fenólicos como flavonoides, quercetina e rutina (CABRAL-MALHEIROS et al., 2010). O objetivo do trabalho foi avaliar a eficácia da incorporação do extrato aquoso de erva-mate como aditivo antioxidante para filmes biodegradáveis a base de amido de mandioca, plastificados com glicerol e reforçados com nanocelulose de coco, assim como, analisar a influência do aditivo nas propriedades mecânicas e de barreiras dos nanobiocompósitos. A ação antioxidante desses nanobiocompósitos ativos foi avaliada através do monitoramento das alterações químicas da embalagem e do produto embalado. Destaca-se que nenhum outro estudo foi encontrado na literatura com nanocristais de celulose incorporados num filme com propriedades ativas (embalagem ativa).

\section{MATERIAL E MÉTODOS}

A metodologia para extração da polpa de celulose da fibra de coco foi adaptada da literatura, utilizando como referência os trabalhos de ROSA et al. (2010) e SAMIR et al. (2005). A polpa de celulose foi submetida ao processo de hidrólise ácida, utilizando $\mathrm{H}_{2} \mathrm{SO}_{4}$ 64\% (Merck, Alemanha) para a obtenção da solução de nanocelulose. Um total de $12 \mathrm{ml}$ de ácido para cada grama de celulose foi submetido à agitação durante $10-15$ minutos $\left(50^{\circ} \mathrm{C}\right)$. Para a análise de microscopia eletrônica de transmissão (TEM), a solução foi misturada em volumes iguais com acetato de uranila a $2 \%$ (UA) (Merck, Alemanha) e $10 \mathrm{~mL}$ da mistura UAnanocelulose foram dispensados em uma grade de cobre, 400 mesh, e deixada em repouso durante 30-60 segundos. A grade foi seca e visualizada em um microscópio eletrônico de transmissão (Fei Morgani, modelo 268D) no modo de campo claro em $80 \mathrm{kV}$, para determinar o comprimento (L), largura (D), relação de aspecto $(\mathrm{L} / \mathrm{D})$ dos nanocristais.

Os nanobiocompósitos foram elaborados por casting, que consistiu no preparo de uma solução filmogênica, em que, inicialmente, foi feito um extrato aquoso de erva-mate, obtido através da percolação de 2 litros de água destilada a $70^{\circ} \mathrm{C}$, sobre o pó de ervamate $\left(20 \%\right.$ de pó de erva-mate, g $\left.100 \mathrm{~g}^{-1}\right)$. Em seguida, dissolveu-se nessa solução o amido de mandioca (Amilogill 1500 cedido pela Cargill Agrícola S.A., produto amiláceo obtido pelo processamento de fécula de mandioca com umidade $11-14 \%$ e $\mathrm{pH} 4,8-6,0)$ e o glicerol (plastificante) $\left(\operatorname{Vetec}^{\circledR}\right.$, Brasil), e, por fim, foi adicionada a solução aquosa de nanocelulose (MACHADO et al., 2010). A solução foi aquecida até a temperatura de gelatinização do amido, $70^{\circ} \mathrm{C}$, sob agitação (VEIGA-SANTOS \& SCAMPARINI, 2004). As soluções $(40 \mathrm{~g})$ foram transferidas para placas de Petri e secas a $35 \pm 2^{\circ} \mathrm{C}$ por 18 horas em estufa (Sterilifer SXCR) com circulação de ar. As formulações A $(6 \%$ de amido, $1,5 \%$ de glicerol, $0,3 \%$ de nanocelulose e $20 \%$ de extrato de erva-mate) e B ( $4,5 \%$ de amido, $0,5 \%$ de glicerol, $0,3 \%$ de nanocelulose e $20 \%$ de extrato de ervamate) foram usadas para embalar azeite de dendê, para investigar o comportamento do aditivo antioxidante contido na embalagem durante armazenamento por 40 dias, em oxidação acelerada $\left(63 \% \mathrm{UR}, 30^{\circ} \mathrm{C}\right)$. Como controle, foi utilizado o azeite de dendê embalado com filme de amido de mandioca sem os aditivos antioxidantes (CA e $\mathrm{CB}$ ), em polietileno de baixa densidade (CP) e sem embalagem (CE), ou seja, 
colocado em uma placa de Petri e submetido às mesmas condições de armazenagem.

As embalagens foram moldadas na forma de sacos retangulares de dimensões $5 \times 2 \mathrm{~cm}\left(10 \mathrm{~cm}^{2}\right)$, seladas utilizando seladora de bancada Sulpack (SM 300 Light) com temperatura contínua e, em cada uma, foram adicionados $10 \mathrm{ml}$ de azeite de dendê, sendo lacradas com a mesma seladora e armazenadas por 40 dias sob oxidação acelerada $\left(63 \% \mathrm{UR}, 30^{\circ} \mathrm{C}\right)$. A estabilidade oxidativa do produto embalado em função da ação antioxidante dos filmes ativos foi monitorada periodicamente, através da determinação do índice de peróxido (IP) do azeite (AOAC Cd 8b-90, 2000) e do teor de polifenóis totais (PT) dos nanobiocompósitos por espectrofotometria (SWAIN \& HILLIS, 1959). Como controle, foi utilizado o azeite de dendê embalado com filme sem os aditivos antioxidantes (CA e $\mathrm{CB}$ ), em polietileno de baixa densidade $(\mathrm{CP})$ e sem embalagem (CE), ou seja, colocado em uma placa de Petri. As análises do monitoramento foram realizadas em triplicata.

A espessura dos nanobiocompósitos ativos pré-acondicionados $\left(60 \% \mathrm{UR}, 25^{\circ} \mathrm{C}\right)$ foi avaliada através da espessura média, de seis medições em posições aleatórias, por meio de micrômetro digital Mitutoyo. As medições de atividade de água (aw), permeabilidade ao vapor de água, umidade e sólidos totais dos nanobiocompósitos ativos foram realizadas conforme metodologia proposta por VEIGA-SANTOS et al. (2005), todas em triplicata. Os ensaios de tração foram realizados em uma máquina universal de ensaios da marca EMIC, modelo DL2000/700, carga máxima de $20 \mathrm{KN}$, seguindo a norma ASTM D-882, velocidade de $12,5 \mathrm{~mm} \mathrm{~min}^{-1}$ e temperatura de $25^{\circ} \mathrm{C}$, em seis corpos de prova para cada amostra.
Os parâmetros do produto embalado e da embalagem foram comparados periodicamente ao longo do armazenamento para um mesmo filme, entre os filmes, assim como com os controles. Para as propriedades de barreira e mecânicas dos nanobiocompósitos foram comparadas as formulações A e B entre si, além de compará-los com os respectivos controles. $\mathrm{Na}$ comparação das variáveis entre filmes e controles, e entre o azeite de dendê embalado em diferentes filmes, foi utilizado o teste de Tukey (ANOVA - Statistica 7.0), usando os efeitos da oxidação lipídica (IP) e a perda dos compostos antioxidantes (PT), ao longo do armazenamento, para comparar o desempenho dos filmes ativos sobre o produto embalado, ao nível de $95 \%$ de confiança. O teste Tukey estabeleceu letras para as médias dos parâmetros avaliados, obtendo-se, resultados com letras diferentes significam que, foram estatisticamente diferentes, e resultados com letras iguais demostram que não foram estatisticamente diferentes.

\section{RESULTADOS E DISCUSSÃO}

Através da análise de TEM, foi possível identificar a presença dos nanocristais de celulose de coco na solução aquosa $\left(0,66 \mathrm{~g} 10 \mathrm{ml}^{-1}\right)$, que consiste principalmente de fibrilas individuais e de alguns agregados (Figura 1A e B). Isso é consistente com os resultados obtidos por SAMIR et al. (2005), LAI-KEEHIM et al. (2002) e ROSA et al. (2010). Os nanocristais de celulose de coco apresentaram comprimentos (L) que variaram de 98 a 430nm e largura (D) de cerca de $6 \mathrm{~nm}$. A relação de comprimento e largura (L/D) apresentou um valor médio de 39, indicando, portanto, grande potencial para serem usados como reforço em

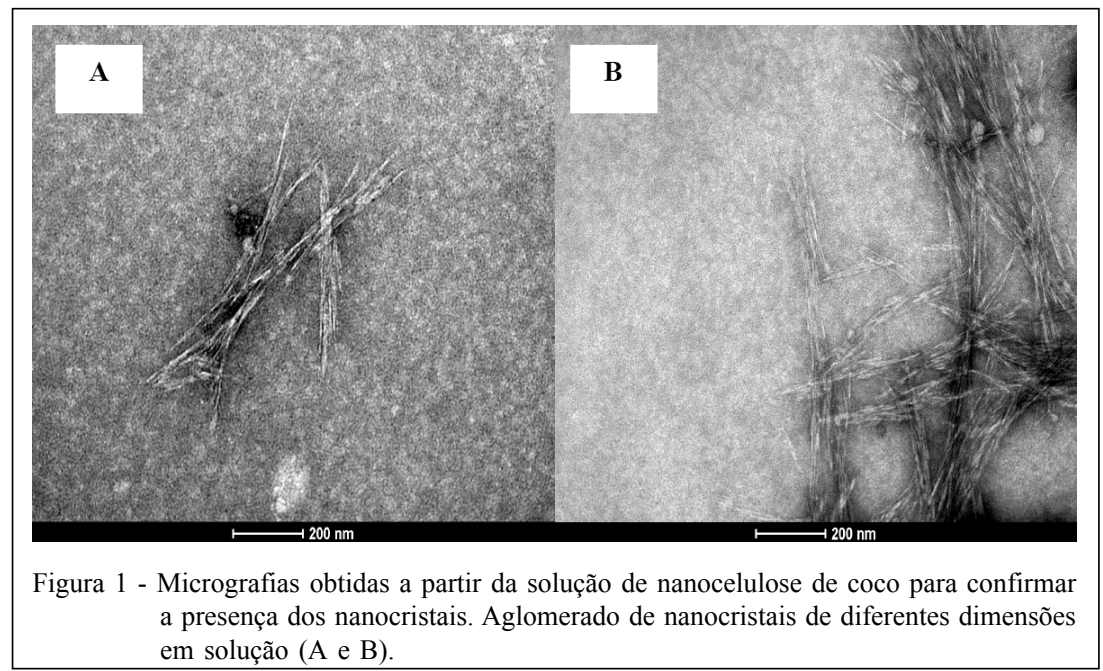

Ciência Rural, v.42, n.11, nov, 2012. 
nanocompósitos (ROSA et al., 2010). Os resultados encontrados neste estudo são semelhantes aos encontrados por outros autores que caracterizaram as dimensões da nanocelulose obtidas de diferentes fontes (BONDESON et al., 2006; SOUZA-LIMA et al., 2003; SAMIR et al., 2005; ELAZZOUZI-HAFRAOUI et al., 2008; ROSA et al., 2010).

$\mathrm{O}$ aditivo, extrato de erva-mate, incorporado aos filmes de amido de mandioca como fonte de compostos ativos, contém concentrações substanciais de PT $\left(139,9 \pm 1,12 \mathrm{mg} \mathrm{g}^{-1}\right)$, com teores dentro dos limites reportados na literatura para essa planta (REIS, 2011; KUSKOSKI et al., 2006). O efeito da incorporação de $20 \%$ de extrato de erva-mate $(\mathrm{m} / \mathrm{v})$ nas formulações Ae $\mathrm{B}$ durante o armazenamento do azeite de dendê nos filmes por 40 dias estão apresentados na tabela 1 . Os teores de PT variaram de 60,72 a 102,70 $\mathrm{mg} \mathrm{g}^{-1}$ para a formulação A e de 61,00 a $101,90 \mathrm{mg} \mathrm{g}^{-1}$ para a formulação B, indicando que ocorrem perdas significativas $(\mathrm{P}>0,05)$ entre o início e final do armazenamento, entretanto, mesmo após esse período, os compostos ativos do aditivo permanecem viáveis nos filmes. Comparando a perda de PT em cada intervalo de armazenamento, nota-se que foi similar entre os diferentes intervalos estudados, sem diferenças significativas $(\mathrm{P}<0,05)$ entre as formulações A e B. Ao final dos 40 dias foi determinada uma perda total de $40 \%$ no conteúdo de PT para as duas formulações. Azeite de dendê também foi embalado com filmes de amido de mandioca ativados com diferentes quantidades de polpa de manga e extrato de erva-mate por REIS (2011), e, após 45 dias de armazenamento, resultou em redução entre 15,0 a 27,7\% nos PT dos filmes, e com filmes com acerola e manga, a redução dos teores de PT foi de 17,80 a 36,12\% (SOUZA et al., 2011).
O IP do azeite de dendê embalado nos filmes contendo $20 \%$ de extrato de erva-mate, e nos filmes sem o aditivo também foi monitorado periodicamente, constatando-se que o azeite embalado nos dois filmes ativos e nos controles (CA, CB, CP e CE) apresentou um aumento gradual do IP, com diferentes intensidades, dependendo da embalagem (Tabela 2).

$\mathrm{O}$ azeite de dendê embalado nos filmes com o aditivo (A e B) apresentou comportamento similar ao longo de 40 dias de armazenamento, com um menor aumento no IP $(\mathrm{P}<0,05)$, quando comparado aos apresentados pelo produto embalado com os quatro controles, indicando a maior eficácia do extrato de ervamate como aditivo antioxidante. $\mathrm{O}$ azeite de dendê embalado nos controles CA e CB sem aditivo também apresentou comportamento semelhante no aumento do IP, indicando assim que o percentual de amido e glicerol não influenciou neste parâmetro. Entretanto, os resultados também indicam que, mesmo sem os aditivos antioxidantes, os filmes de amido de mandioca têm um efeito protetor maior da oxidação do azeite $(\mathrm{P}<0,05)$, quando comparados aos filmes de PEBD (CP). Esses resultados mostram que os filmes de amido de mandioca com nanocelulose podem representar uma barreira mais eficaz ao oxigênio do que os filmes de PEBD. A presença dos nanocristais de celulose na matriz é responsável por reduzir a permeabilidade a gases e ao vapor de água (Tabela 3), levando a alterações na estrutura dos nanobiocompósitos. Esse efeito é atribuído à presença de fortes interações de hidrogênio entre os nanocristais, que possuem elevada cristalinidade, com a matriz hidrofílica de amido e o glicerol, agindo assim como uma barreira e diminuindo os espaços livres na matriz polimérica, dificultando a passagem de vapores (MÜLLER et al., 2008; BECK et al., 2005). Dentre os

Tabela 1 - Teores e reduções periódicas e totais dos polifenóis totais (PT) dos filmes durante o armazenamento do azeite de dendê por até 40 dias.

\begin{tabular}{|c|c|c|c|c|c|c|}
\hline \multirow{2}{*}{ Filme } & \multicolumn{5}{|c|}{ 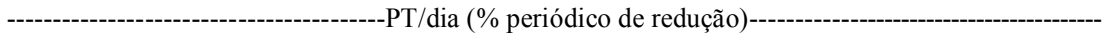 } & \multirow{2}{*}{ Redução Total $0-40$} \\
\hline & 0 & 10 & 20 & 30 & 40 & \\
\hline A & $\begin{array}{c}102,70 \pm 1,23^{\mathrm{a}, \mathrm{A}} \\
(0)^{\mathrm{b}, \mathrm{A}}\end{array}$ & $\begin{array}{c}94,80 \pm 1,54^{\mathrm{a}, \mathrm{B}} \\
(7,69)^{\mathrm{b}, \mathrm{B}}\end{array}$ & $\begin{array}{c}81,90 \pm 1,17^{\mathrm{a}, \mathrm{C}} \\
(8,64)^{\mathrm{b}, \mathrm{B}, \mathrm{C}}\end{array}$ & $\begin{array}{c}73,70 \pm 1,14^{\mathrm{a}, \mathrm{D}} \\
(8,99)^{\mathrm{b}, \mathrm{C}}\end{array}$ & $\begin{array}{c}60,72 \pm 1,88^{\mathrm{a}, \mathrm{E}} \\
(8,24)^{\mathrm{b}, \mathrm{B}, \mathrm{C}}\end{array}$ & $\begin{array}{l}41,98^{\mathrm{a}} \\
(40,87)^{\mathrm{b}}\end{array}$ \\
\hline $\mathrm{B}$ & $\begin{array}{c}101,90 \pm 1,19^{\mathrm{a}, \mathrm{A}} \\
(0)^{\mathrm{b}, \mathrm{A}}\end{array}$ & $\begin{array}{c}93,70 \pm 1,37^{\mathrm{a}, \mathrm{B}} \\
(8,05)^{\mathrm{b}, \mathrm{B}}\end{array}$ & $\begin{array}{c}82,10 \pm 1,65^{\mathrm{a}, \mathrm{C}} \\
(8,76)^{\mathrm{b}, \mathrm{B}, \mathrm{C}}\end{array}$ & $\begin{array}{c}74,10 \pm 1,22^{\mathrm{a}, \mathrm{D}} \\
(9,25)^{\mathrm{b}, \mathrm{C}}\end{array}$ & $\begin{array}{c}61,00 \pm 1,13^{\mathrm{a}, \mathrm{E}} \\
\quad(8,23)^{\mathrm{b}, \mathrm{B}}\end{array}$ & $\begin{array}{l}40,90^{\mathrm{a}} \\
(40,14)^{\mathrm{b}}\end{array}$ \\
\hline
\end{tabular}

PT em $\mathrm{mg} \mathrm{g}^{-1}$.

A: Formulação com $6 \%$ de amido, $1,5 \%$ de glicerol, $0,3 \%$ de nanocelulose e $20 \%$ de extrato de erva-mate.

B: Formulação com $4,5 \%$ de amido, $0,5 \%$ de glicerol, $0,3 \%$ de nanocelulose e $20 \%$ de extrato de erva-mate.

Médias seguidas da mesma letra maiúscula na mesma linha e letra minúscula iguais na mesma coluna não diferem entre si pelo teste de Tukey (5\%). 
Desenvolvimento e avaliação da eficácia de filmes biodegradáveis de amido de mandioca com nanocelulose...

Tabela 2 - Índice de peróxidos (IP) do azeite de dendê embalado nos filmes por até 40 dias de armazenamento e percentual de aumento durante o período total.

\begin{tabular}{|c|c|c|c|c|c|c|}
\hline \multirow{2}{*}{ Filme } & \multicolumn{5}{|c|}{ 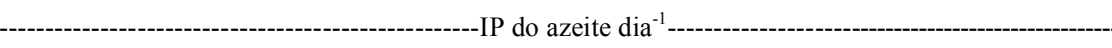 } & \multirow{2}{*}{ Aumento IP 0-40 (\%) } \\
\hline & 0 & 10 & 20 & 30 & 40 & \\
\hline A & $2,90 \pm 0,09^{\mathrm{a}, \mathrm{A}}$ & $3,14 \pm 0,02^{\mathrm{a}, \mathrm{B}}$ & $7,72 \pm 0,11^{\mathrm{a}, \mathrm{C}}$ & $10,78 \pm 0,66^{\mathrm{a}, \mathrm{D}}$ & $35,41 \pm 0,93^{\mathrm{a}, \mathrm{E}}$ & $1121,94^{\mathrm{a}, \mathrm{F}}$ \\
\hline $\mathrm{B}$ & $2,90 \pm 0,03^{\mathrm{a}, \mathrm{A}}$ & $3,16 \pm 0,08^{\mathrm{a}, \mathrm{B}}$ & $7,83 \pm 0,13^{\mathrm{a}, \mathrm{C}}$ & $10,80 \pm 0,61^{\mathrm{a}, \mathrm{D}}$ & $35,57 \pm 0,87^{\mathrm{a}, \mathrm{E}}$ & $1227,99^{\mathrm{a}, \mathrm{F}}$ \\
\hline $\mathrm{CA}$ & $2,90 \pm 0,04^{\mathrm{a}, \mathrm{A}}$ & $5,09 \pm 0,11^{\mathrm{b}, \mathrm{B}}$ & $14,01 \pm 0,35^{\mathrm{a}, \mathrm{C}}$ & $34,90 \pm 0,80^{\mathrm{b}, \mathrm{D}}$ & $60,09 \pm 0,78^{\mathrm{b}, \mathrm{E}}$ & $2075,78^{\mathrm{b}, \mathrm{F}}$ \\
\hline $\mathrm{CB}$ & $2,90 \pm 0,08^{\mathrm{a}, \mathrm{A}}$ & $5,03 \pm 0,03^{\mathrm{b}, \mathrm{B}}$ & $13,98 \pm 0,33^{\mathrm{b}, \mathrm{C}}$ & $33,95 \pm 0,73^{\mathrm{b}, \mathrm{D}}$ & $59,02 \pm 0,83^{\mathrm{b}, \mathrm{E}}$ & $2038,08^{\mathrm{b}, \mathrm{F}}$ \\
\hline $\mathrm{CP}$ & $2,90 \pm 0,04^{\mathrm{a}, \mathrm{A}}$ & $11,79 \pm 0,27^{\mathrm{c}, \mathrm{B}}$ & $33,35 \pm 0,47^{\mathrm{c}, \mathrm{C}}$ & $82,79 \pm 0,97^{\mathrm{c}, \mathrm{D}}$ & $97,00 \pm 0,98^{\mathrm{c}, \mathrm{E}}$ & $3347,17^{\mathrm{c}, \mathrm{F}}$ \\
\hline $\mathrm{CE}$ & $2,89 \pm 0,02^{\mathrm{a}, \mathrm{A}}$ & $14,06 \pm 0,33^{\mathrm{d}, \mathrm{B}}$ & $44,44 \pm 0,23^{\mathrm{d}, \mathrm{C}}$ & $94,49 \pm 0,89^{\mathrm{d}, \mathrm{D}}$ & $115,56 \pm 0,93^{\mathrm{d}, \mathrm{E}}$ & $3993,08^{\mathrm{d}, \mathrm{F}}$ \\
\hline
\end{tabular}

IP em meq $100 \mathrm{~g}^{-1}$

CA e CB: Formulações A e B sem o extrato de erva-mate.

CP: embalagem de PEBD.

CE: sem embalagem.

Médias seguidas da mesma letra maiúscula na mesma linha e letra minúscula iguais na mesma coluna não diferem entre si pelo teste de Tukey $(5 \%)$.

controles avaliados, o azeite exposto (CE) apresentou o maior aumento do IP. Outros estudos também relatam a ampla ação antioxidante de filmes biodegradáveis formulados com amido de mandioca e aditivos naturais (polpa de manga, acerola, dendê) como compostos ativos antioxidantes (GRISI et al., 2008; SOUZA et al., 2011; REIS et al., 2011).

A avaliação do efeito da incorporação do extrato de erva-mate sobre as características mecânicas e de barreira dos nanobiocompósitos estão relacionados na tabela 3 . A incorporação do extrato de erva-mate na matriz polimérica de amido, reforçada pela adição de nanocelulose de coco, não alterou significativamente $(\mathrm{P}>0,05)$ as propriedades de barreira dos nanobiocompósitos. O mesmo comportamento foi observado para as propriedades mecânicas, ou seja, não houve alteração significativa $(\mathrm{P}>0,05)$ no módulo, tração e deformação dos nanobiocompósitos. Portanto, os nanobiocompósitos passaram a ter uma vantagem adicional de fornecer a propriedade antioxidante, sem alterações nas propriedades de barreira e mecânicas. Os dois filmes, A e B, por serem formulados a partir de diferentes concentrações de amido e glicerol, quando comparados entre si, apresentaram diferenças significativas $(\mathrm{P}<0,05)$ nas propriedades mecânicas. $\mathrm{O}$ filme $\mathrm{B}$ apresentou os maiores valores para módulo de Young $(471,10 \pm 4,65 \mathrm{MPa})$ e tensão máxima $(13,96 \pm 0,98 \mathrm{MPa})$ e, em contrapartida, um menor percentual de deformação $(5,98 \pm 0,13 \%)$, quando comparado com a formulação A (Tabela 3). O maior percentual de deformação encontrado para o filme A $(57,09 \pm 0,91 \%)$ está relacionado ao maior teor de glicerol presente nessa formulação. Entretanto, diferenças significativas $(\mathrm{P}>0,05)$ não foram observadas quando comparadas as propriedades de barreira dos dois filmes. Para o parâmetro espessura, foram observadas

Tabela 3 - Parâmetros das propriedades de barreiras e mecânicas dos filmes A e B e dos controles (CA e CB).

\begin{tabular}{|c|c|c|c|c|c|c|c|c|}
\hline Filme & $\mathrm{E}$ & aw & ST & $\mathrm{U}$ & PVA. $10^{-8}$ & $\mathrm{E}$ & $\sigma$ & $\mathrm{E}$ \\
\hline $\mathrm{A}$ & $0,15 \pm 0,02^{\mathrm{a}}$ & $0,69 \pm 0,06^{\mathrm{a}}$ & $82,01 \pm 2,01^{\mathrm{a}}$ & $17,99 \pm 1,18^{\mathrm{a}}$ & $5,95 \pm 0,76^{\mathrm{a}}$ & $33,99 \pm 1,06^{\mathrm{a}}$ & $3,21 \pm 0,15^{\mathrm{a}}$ & $57,09 \pm 0,91^{\mathrm{a}}$ \\
\hline $\mathrm{CA}$ & $0,15 \pm 0,03^{\mathrm{a}}$ & $0,69 \pm 0,10^{\mathrm{a}}$ & $81,22 \pm 1,85^{\mathrm{a}}$ & $18,78 \pm 1,49^{\mathrm{a}}$ & $5,97 \pm 0,86^{\mathrm{a}}$ & $34,19 \pm 1,13^{\mathrm{a}}$ & $3,22 \pm 0,41^{\mathrm{a}}$ & $56,18 \pm 1,20^{\mathrm{a}}$ \\
\hline B & $0,10 \pm 0,03^{b}$ & $0,64 \pm 0,08^{\mathrm{a}}$ & $83,70 \pm 1,85^{\mathrm{a}}$ & $16,30 \pm 1,10^{\mathrm{b}}$ & $5,93 \pm 0,81^{\mathrm{a}}$ & $471,10 \pm 4,65^{\mathrm{b}}$ & $13,96 \pm 0,98^{\mathrm{b}}$ & $5,98 \pm 0,13^{\mathrm{b}}$ \\
\hline $\mathrm{CB}$ & $0,10 \pm 0,02^{\mathrm{b}}$ & $0,64 \pm 0,07^{\mathrm{a}}$ & $84,50 \pm 1,75^{\mathrm{a}}$ & $15,50 \pm 1,22^{b}$ & $5,94 \pm 0,93^{\mathrm{a}}$ & $470,90 \pm 9,07^{\mathrm{b}}$ & $14,09 \pm 1,22^{b}$ & $6,01 \pm 0,43^{\mathrm{b}}$ \\
\hline
\end{tabular}

E (espessura - mm); aw (atividade de água); ST (sólidos totais - \%); Umidade (\%); PVA (permeabilidade ao vapor de água $\mathrm{gH}_{2} \mathrm{O} . \mu \mathrm{m} / \mathrm{m}^{2}$.h.mmHg), E (Módulo de Young - MPa); $\sigma$ (Tensão Máxima - MPa); $\varepsilon$ (Deformação - \%).

A: Formulação com $6 \%$ de amido, $1,5 \%$ de glicerol, $0,3 \%$ de nanocelulose e $20 \%$ de extrato de erva-mate.

B: Formulação com 4,5\% de amido, $0,5 \%$ de glicerol, $0,3 \%$ de nanocelulose e $20 \%$ de extrato de erva-mate.

CA e CB: Formulações A e B sem o extrato de erva-mate.

Médias seguidas da mesma letra na mesma coluna não diferem entre si pelo teste de Tukey (5\%).

Ciência Rural, v.42, n.11, nov, 2012. 
diferenças significativas $(\mathrm{P}<0,05)$ entre $\mathrm{A}$ e $\mathrm{B}$, sendo que o filme $\mathrm{A}$ apresentou um maior valor médio $(0,15 \pm 0,02 \mathrm{~mm})$, devido ao maior conteúdo de amido presente nesta formulação. Portanto, as duas formulações resultam em filmes que podem ser empregados para diferentes aplicações, independente do aditivo incorporado, já que diferem em relação ao módulo, tensão e deformação. Ressalta-se ainda que as ótimas propriedades mecânicas apresentadas pelos filmes (A, B, CA e CB) estão relacionadas à presença dos nanocristais de celulose de coco, como relatado por outros autores que também incorporaram nanocristais de celulose de diferentes fontes em matrizes poliméricas biodegradáveis (WANG et al., 2006; CAO et al., 2008). SOUZA et al. (2012) relatam valores bastante inferiores para a tensão $(1,85$ a $4,81 \mathrm{MPa}$ ) de filmes de amido sem a presença de nanocristais de celulose. Além disso, os valores de resistência à tração encontrados no presente estudo são semelhantes aos relatados para filmes sintéticos de polietileno de baixa densidade (PEBD), que variam de 6,9 a 16MPa (COUTINHO et al., 2003). O efeito da melhoria das propriedades mecânicas e de barreira dos filmes elaborados neste estudo pode ser atribuído ao fenômeno de percolação mecânico dos nanocristais de celulose e à formação de uma rede contínua da nanocelulose, ligada por interações de hidrogênio, devido a uma boa dispersão dos nanocristais na matriz, o que indica uma boa interação entre os componentes do filme, tornando-os competitivos com o PEBD, independente da ação antioxidante, com as vantagens adicionais de serem totalmente biodegradáveis.

\section{CONCLUSÃO}

Os resultados apresentados neste estudo evidenciam que o desenvolvimento de filmes antioxidantes com extrato de erva-mate como fonte de compostos ativos incorporados à matriz de amido de mandioca plastificada com glicerol é viável e pode ser aplicada como embalagem para evitar a oxidação de produtos lipídicos. Além disso, a incorporação desse aditivo natural não altera as propriedades de barreira e mecânicas dos nanobiocompósitos, permitindo assim a sua utilização como embalagem ativa antioxidante com propriedades mecânicas melhoradas e diferenciadas, devido à nanocelulose de coco e aos diferentes teores da matriz e de plastificante.

\section{AGRADECIMENTOS}

Ao Programa NANOBIOTEC - Coordenação de Aperfeiçoamento de Pessoal de Nível Superior (CAPES) ao qual esta pesquisa está vinculada, e à Fundação de Amparo à Pesquisa do Estado da Bahia (Fapesb) pelo apoio financeiro da bolsa de pesquisa.

\section{REFERÊNCIAS}

AOAC (ASSOCIATION OF OFFICIAL ANALYTICAL CHEMISTS). Official methods of analysis Cd 8b-90. Washington, 2000. 1141p.

ASTM (AMERICAN SOCIETY FOR TESTING AND MATERIALS). Standard test method for tensile properties of thin plastic sheeting ASTM D882-00. Philadelphia, 2001. 9p.

BONDESON, D. et al. Optimization of the isolation of nanocrystals from microcrystalline cellulose by acid hydrolysis. Cellulose, v.13, p.171-180, 2006. Disponível em: <http:// www.springerlink.com/content/mv5075176w121523/>. Acesso em: 09 out. 2011. doi: 10.1007/s10570-006-9061-4.

BECK, C.S. et al. Effect of reaction conditions on the properties and behavior of wood cellulose nanocrystal suspensions. Biomacromolecules, v.6, p.1048-1054, 2005. Disponível em: <http://pubs.acs.org/doi/abs/10.1021/bm049300p>. Acesso em: 12 out. 2011 . doi: 10.1021/bm049300p.

CABRAL-MALHEIROS, G. et al. O tempo e o tipo de embalagem sobre a erva-mate tipo chimarrão durante armazenagem em condições ambientais. Ciência Rural, v.40, n.3, p.654-660, 2010. Disponível em: <http://dx.doi.org/ 10.1590/S0103-84782010005000028>. Acesso em: 14 out. 2011. doi: 10.1590/S0103-84782010005000028.

CAO, X. et al. Green composites reinforced with hemp nanocrystals in plasticized starch. Journal of Applied Polymer Science, v.109, p.3804-3810, 2008. Disponível em: <http://onlinelibrary.wiley.com/doi/10.1002/app.28418/ full $>$. Acesso em: 10 out. 2011. doi: 10.1002/app.28418.

COUTINHO, F.M.B. et al. Polietileno: principais tipos, propriedades e aplicações. Polímeros, v.13, n.1, p.1-13, 2003. Disponível em: $<$ http://dx.doi.org/10.1590/S0104-14282003000100005>. Acesso em: 03 set. 2011. doi: 10.1590/S0104-14282003000100005.

ELAZZOUZI-HAFRAOUI, S. et al. The shape and size distribution of crystalline nanoparticles prepared by acid hydrolysis of native cellulose. Biomacromolecules, v.9, p.57-65, 2008.

FUKUMOTO, L.R.; MAZZA, G. Assessing antioxidant and prooxidant activities of phenolic compounds. Journal of Agricultural and Food Chemistry, v.48, n.8, p.3597-3604, 2000. Disponível em: <http://pubs.acs.org/doi/abs/10.1021/ jf000220w>. Acesso em: 25 set. 2011. doi: 10.1021/jf000220w.

GRISI, C.V.B. et al. Evaluation of the viability of incorporating natural antioxidants in bio-based packagings. Nova Science Publishers - Food Chemistry Research Developments, NY 11788, v.1, p.1-11, 2008.

LAI-KEE-HIM, H. et al. In vitro versus in vivo cellulose microfibrils from plant primary wall synthases: Structural differences. Journal of Biological Chemistry, v.277, n.40, p.36931-36939, 2002. Disponível em: <http://www.jbc.org/ content/277/40/36931.long $>$. Acesso em: 14 out. 2011. doi: $10.1074 /$ jbc.M203530200.

LOULI, V. et al. Recovery of phenolic antioxidants from wine industry by-products. Bioresource Technology, v.92, p.201208, 2004. Disponível em: <http://dx.doi.org/10.1016/ j.biortech.2003.06.002>. Acesso em: 09 out. 2011. doi: 10.1016/j.biortech.2003.06.002. 
LU, Y. et al. Biocomposites of plasticized starch reinforced with cellulose crystallites from cottonseed linter. Macromolecular Bioscience, v.5, p.1101-1107, 2005. Disponível em: <http://onlinelibrary.wiley.com/doi/10.1002/ mabi.200500094>. Acesso em: 14 out. 2011. doi: 10.1002/ mabi.200500094.

MACHADO, B.A.S. et al. Processo para a preparação de nanobiocompósitos tipo filmes flexíveis reforçados com nanopartículas de celulose tipo nanowhiskers. Patente protocolizada no INPI, 2010, Br.

MALI, S. et al. Filmes de amido: produção, propriedades e potencial de utilização. Ciências Agrárias, v.31, n.1, p.137156, 2010. Disponível em: <http://www.uel.br/revistas/uel/ index.php/semagrarias/article/view>. Acesso em: 30 set. 2011.

MÜLLER, C.M.O. et al. Evaluation of the effects of glycerol and sorbitol concentration and water activity on the water barrier properties of cassava starch films through a solubility approach. Carbohydrate Polymers, v.72, p.82-87, 2008. Disponível em: <http://dx.doi.org/10.1016/j.carbpol.2007.07.026>. Acesso em: 29 set. 2011. doi: 10.1016/j.carbpol.2007.07.026.

REIS, L.C.B. Formulação e caracterização de filmes biodegradáveis de fécula de mandioca e incorporados com polpa de manga e extrato de erva-mate, e seu efeito na preservação de alimentos. 2011. 151f. Dissertação (Mestrado em Ciências de Alimentos) - Faculdade de Farmácia, Universidade Federal da Bahia, Ba.

ROSA, M.F. et al. Cellulose nanowhiskers from coconut husk fibers: Effect of preparation conditions on their thermal and morphological behavior. Carbohydrate Polymers, v.81, p.83-92, 2010. Disponível em: <http://dx.doi.org/10.1016/ j.carbpol.2010.01.059>. Acesso em: 29 set. 2011. doi: 10.1016/ j.carbpol.2010.01.059

SAMIR, M.A.S.A. et al. Review of recent research into cellulosic whiskers, their properties and their application in nanocomposite field. Biomacromolecules, v.6, p.612-626, 2005. Disponível em: <http://pubs.acs.org/doi/abs/10.1021/ bm0493685>. Acesso em: 27 set. 2012. doi: 10.1021/ bm0493685.

SILVA, R. et al. Aplicações de fibras lignocelulósicas na química de polímeros e em compósitos. Química Nova, v.32, n.3, p.661-671, 2009. Disponível em: <http://dx.doi.org/10.1590/ S0100-40422009000300010>. Acesso em: 12 out. 2011. doi: 10.1590/S0100-40422009000300010.

SILVA, L.T. Desenvolvimento e avaliação de embalagens biodegradáveis com ação antioxidante contendo café e cacau. 2009. 148f. Dissertação (Mestrado em Ciência de Alimento) Faculdade de Farmácia, Universidade Federal da Bahia, Ba.
SOUZA, C.O. et al. Mango and Acerola pulps as antioxidant additives in Cassava starch bio-based film. Journal of Agricultural and Food Chemistry, v.59, n.6, p.2248-2254, 2011. Disponível em: <http://pubs.acs.org/doi/abs/10.1021/ jf1040405>. Acesso em: 12 out. 2011. doi: 10.1021/jf104040.

SOUZA, C.O. et al. Estudo comparativo da caracterização de filmes biodegradáveis de amido de mandioca contend polpas de manga e de acerola. Química Nova, v.35, n.2 p.262-267, 2012. Disponível em: $<\mathrm{http}: / / \mathrm{dx}$.doi.org/10.1590/S010040422012000200006>. Acesso em: 01 jan. 2012. doi: 10.1590/ S0100-40422012000200006

SOUZA-LIMA, M.M. et al. Translational and rotational dynamics of rodlike cellulose whiskers. Langmuir, v.19, p.24-29, 2003.

SWAIN, T.; HILLIS, W.E. The phenolic constituents of Prunus doméstica: the quantitative analysis of phenolic constituents. Journal of the Science of Food and Agriculture, v.10, p.63-68, 1959. Disponível em: <http://pubs.acs.org/doi/abs/ 10.1021/la020475z>. Acesso em: 24 set. 2011. doi: 10.1021/ $1 \mathrm{a} 020475 \mathrm{z}$.

VEIGA-SANTOS, P. et al. Mechanical properties, hydrophilicity and water activity of starch-gum films: Effect of additives and deacetylated xanthan gum. Food Hydrocolloids, v.19, p.341-349, 2005. Disponível em: <http:/ /dx.doi.org/10.1016/j.foodhyd.2004.07.006>. Acesso em: 11 out. 2011. doi:10.1016/j.foodhyd.2004.07.006.

VEIGA-SANTOS, P.; SCAMPARINI, A.R.P. Indicador irreversível de temperatura utilizando carboidratos. PI0403610-7 A2, patente protocolizada no INPI, 2004

VEIGA, P.S.; DRUZIAN, J.I. Absorvedores de umidade/ antiembaçantes biodegradáveis à base de amido/fécula para serem aplicados em produtos de higiene pessoal, alimentícios ou farmacêuticos. Patente protocolizada no INPI, 2007.

WANG, Y. et al. Effects of cellulose whiskers on properties of soy protein thermoplastics. Macromolecular Bioscience, v.6, p.524-531, 2006. Disponível em: <http:// onlinelibrary.wiley.com/doi/10.1002/mabi.200600034>. Acesso em: 13 out. 2011. doi: 10.1002/mabi.200600034.

WANG, Y. et al. Role of starch nanocrystals and cellulose whiskers in synergistic reinforcement of waterborne polyurethane. Carbohydrate Polymers, v.80, p.665-671, 2010. Disponível em: <http://dx.doi.org/10.1016/ j.carbpol.2009.10.043>. Acesso em: 13 out. 2011. doi: 10.1016/j.carbpol.2009.10.043. 